\title{
A Novel Predictive Scheme for Confirming State of Bipolar Disorder using Recurrent Decision Tree
}

\author{
Yashaswini K.A, Dr. Aditya Kishore Saxena \\ Dept of Computer Science and Engineering \\ School of Engineering, Presidency University \\ Bangalore, India
}

\begin{abstract}
Bipolar disorder is one of the most challenging illnesses where medical science is still struggling to achieve its landmark therapies. After reviewing existing prediction-based approaches towards investigating bipolar disorder, it is noted that existing approaches are more or less symptomatic and relates depression as sadness. It implies various theories that don't consider many precise indicators of confirming bipolar disorder. Therefore, this manuscript presents a novel framework capable of treating the dataset of depression and fine-tune it appropriately to subject it further to a machine learning-based predictive scheme. The proposed system subjects its dataset for a series of data cleaning operations followed by data preprocessing using a standard scale of rating bipolar level. Further usage of feature engineering and correlation analysis renders more contextual inference towards its statistical score. The proposed system also introduces a Recurrent Decision Tree that further contributes towards the predictive outcome of bipolar disorder. The outcome obtained showcases that the proposed scheme performs better than the conventional decision tree.
\end{abstract}

Keywords-Bipolar disorder; depression; recurrent neural network; decision tree; prediction; sadness

\section{INTRODUCTION}

The proposed paper is about performing prediction towards confirming if the subject is suffering from bipolar disorder on the basis of analysis of their mood-based statistics. Mood disorders are disturbing mental disorders that place an enormous burden on individuals, health care systems, and economies. The two most common mood swing disorders are depression and bipolar disorder (BD) [1-2]. Every year approximately $15 \%$ of the global population experiences depression, and $4 \%$ experiences bipolar disorder during their lifetime. An individual suffering from depression has marked symptoms of sadness, feelings of emptiness, anxiety, sleep disturbances, and a general lack of motivation and interest in activities [3]. Additional symptoms may include feelings of guilt or worthlessness, decreased energy, difficulty concentrating, suicide, and psychotic behavior. The severity of depression depends on multiple factors such as type of symptoms, duration, and its impact on the individual's social and occupational life. An additional serious mental illness, bipolar disorder, is also associated with depression [4]. An important difference between unipolar depression and bipolar disorder is that episodes of mania characterize the latter. This is characterized by inflated self-esteem, impulsive behavior, increased activity, decreased sleep, and goal-directed behavior
[5]. Both conditions are inherited and are linked to genetic susceptibility to environmental elements, causing disruptions to internal biological and emotional states. BD symptoms are also associated with physical health problems, side effects, and social factors, and alcohol and drug abuse, which may also contribute to BD symptoms in all individuals [6]. Therefore, it is evident that increasing stress and unhealthy routine in our modern lifestyle result in BD, mood disorders, depression, and abnormalities. The proposed study focuses on predicting the status of $\mathrm{BD}$ in the next frame. However, it is quite very challenging to diagnose BD types in their early stages due to recurrent depressive episodes. With the advent of modern technologies such as artificial intelligence, machine learning, and other data-driven approaches, many research studies have explored the issue of $\mathrm{BD}$ and its countermeasure [7-8]. However, the analysis of the data streams generated from psychopathology poses a huge challenge due to its complex nature of episodic and temporal characteristics [9]. The studies have shown that data quality has a significant impact on learning or predictive models. This data type requires effective data modeling from the feature engineering viewpoint that exploits distinctive features [10]. Another problem is the selection of suitable data-driven approaches and learning models. Since there are various machine learning (ML) models, each has its own advantages and disadvantages. The selection of a suitable model depends on the dataset characteristics, problem context, model parameters, and configuration. The existing approaches have not considered this factor and adopted a stock ML function lacking additional features and modification.

Therefore, the proposed study address this problem using a unique mechanism of machine learning. The objectives defined for this purpose are as follows: i) to introduces an effective data modeling for feature analysis on bipolar disorder, ii) to present a novel neural network model for predicting the accurate state of bipolar disorder in patients, iii) to determine a suitable feature descriptors based on subjective observations and the clinical rating scales, and iv) to use the feature obtained for bipolar disorder prediction with respect to episodic and temporal, and v) to deploy a recurrent neural decision tree network for processing both episodic and temporal data. The remaining section of this paper is organized as follows: Section II presents the review of literature carried out in the context of bipolar disorder detection and prediction; Section III highlights the research gap identified based on the literature review; Section IV introduces the proposed system and 
methodology adopted; Section V discusses the implementation procedure; Section VI presents analysis of the proposed model while Section VII presents result discussion and performance analysis. Finally, overall work is concluded in Section VIII.

\section{RELATED WORK}

This section provides a brief analysis and review of existing research work in the context of bipolar disorder (BD) prediction in patients. The study also highlights the significant research gaps based on the review analysis.

Millions of people worldwide suffer from BD, which raises serious life-threatening concerns. Many data-driven approaches were introduced in the context of early diagnosis to provide timely treatment. The work carried out by Ceccarelli, and Mahmoud [11] has suggested a multimodal scheme to identify mental disorders from multimedia feeds. This scheme employs a recurrent neural network ( $\mathrm{RNN}$ ) developed based on a fusion technique that considers temporal information in the training phase. The study outcome claims to offer better predictive outcomes than the existing approaches, emphasizing precise modeling of temporal features. In the work of Morla et al. [12], optical coherence tomography (OCT) data is analyzed with different machine learning approaches (ANN) to benefit the diagnosis of $\mathrm{BD}$ in the early stage. The BD patients are identified based on the predive outcome of retinal thinning and thickness of the particular area. However, the study needs an extensive analysis to prove and validate the effectiveness of the presented scheme.

Apart from using OCT data, electroencephalogram (EEG) also provides crucial features to enhance the clinical diagnosis of bipolar disorder. In the study of Sotos et al. [13], the authors have collected EEG data, an extreme applied gradient boosting (XGB) technique to identify BD patients. The authors have also implemented other machine learning classifiers such as knearest neighbors (KNN), decision tree (DT), Naïve Bayes (NB), and support vector machine (SVM) for the comparative assessment. The study claims that XGB outperforms other machine learning classifiers regarding the accuracy, recall, and precision. However, the presented model is not scalable; it does not perform well on sparse data samples and is often prone to outliers. Chen et al. [14] also tried to explore the effectiveness of artificial intelligence with the neuroimaging modalities. The authors have used magnetic resonance images (MRI) to differentiate schizophrenia patients in this study. A discriminative analysis considers coarse-to-fine feature selection to extract the differences between different samples. In this model, SVM is implemented as a predictive model. Sun et al. [15] have utilized Single Nucleotide Polymorphism to obtain features associated with genetic markers. Further, these markers are combined with Convolutional Neural Network $(\mathrm{CNN})$ for recognizing BP patients. The outcome shows that the model has achieved only a $79 \%$ percentage recognition rate and claimed to offer excellent performance.

The deep learning approach is also used in Li et al. [16] for automated diagnosis of BD, psychotic symptoms, and healthy controls. The authors have considered MRI data and constructed CNN to classify the gray matter density images. The outcome shows that this study has achieved an accuracy of
99\% and provides the clinical basis for disease diagnosis and treatment. Though the model can extract features automatically, it requires precise modeling and selection of the layers. Also, the presented model suffers from huge computational complexity and is prone to overfitting and exploiting gradient. Similarly, a more complex learning model can be seen in Huang et al. [17], which is based on the joint approach of CNN and RNN to identify short-term mood disorders. In this work, speech responses were fed to $\mathrm{CNN}$, which provides an emotion profile. Further, RNN is applied to emotion profiles to determine the temporal relationship in emotion profiles to detect mood swings. The work carried out $\mathrm{Hu}$ et al. [18] adopted audio samples, and the RNN model captured temporal patterns from the sequence data samples. The authors have also used the triplet loss function to model discriminative characteristics of the BD severity. The performance analysis is carried out based on the audio/visual emotion challenge dataset. The outcome suggests the effectiveness of the proposed model. The work carried out by Matsubara et al. [19] focuses on the issue of overfitting and extracting a non-relevant feature for the detection of mood swing disorder. The authors have introduced the deep neural generative system in conjunction with Bayes' rules to determine the subsequent probability of the patient state from the given imaging data.

The work of Cigdem et al. [20] has investigated the impacts of covariates on the classification of BD using structural MRI. Voxel-based morphometry is utilized to assess the morphological differences between BD patients and healthy controls. Fitrati et al. [21] used a backpropagation mechanism to detect the $\mathrm{BD}$ using a screening questionnaire. The application of the association rule is considered in the study of Castro et al. [22] to determine the relationship between premenstrual dysphoric disorder and BD. The presented technique is evaluated on a dataset of a cohort of 1099 women. A hypothetical study is conducted in Buttenbender et al. [23]. The authors have considered data generated from mobile devices to identify neuropsychiatric disorders' behavior and support decisions. The entire procedure is carried out in three steps. First, neuropsychiatric disorders are distinguished using dispersion dataset, contextual information based epidemiological profile extraction, and finally, the model is validated against time complexity and scalability. Nunes et al. [24] have suggested a hybrid model that integrates modern and consistent tools using the multicriteria methodology to facilitate the early diagnosis of various psychological disorders, including BD. This study offers a good decision support system, but it is limited in scalability. The work towards the classification of BD type- 1 and BD type- 2 using data modeling technique is carried out by Lee et al. [25]. The authors have built a complementary diagnostic classifier and Gene Ontology (GO) to support the decision-making and functional analysis. Villasanti et al. [26] have presented mathematical modeling based on the differential equation to analyze mood dynamics. The work of Huang et al. [27] focuses on detecting uni-polar and BD based on the pattern obtained from elicited speech. A latent affective structure model and the spectral clustering technique for the data modeling are developed. For early diagnosis and prospective examinations, Demir et al. [28] attempted to determine the distinct features of brain white 
matter in BD. The work of Yashaswini et al. [29] introduces a mechanism of modelling of transition dynamic of mixed mood for controlling the behavioral stimulation of a subject. The study contributes to attain fine information related to dynamics of mood transition.

\section{RESEARCH PROBLEM}

The shortcomings of existing schemes in detecting and distinguishing human bipolar disorder have prompted researchers to attempt data-driven and predictive systems modeling. Based on a literature review, it has been explored that there are still substantial issues in existing approaches that need to be improved with a unique implementation strategy despite many research efforts. This section highlights the critical issues and research gaps identified to benefit the core research area.

- Lack of effective exploratory analysis: It has been identified that much of the existing research works suffers in determining meaningful information from complex time series data. The implications of their implementation and execution are uncertain and limited to explore how ML can be leveraged for patient care.

- Lack of effective benchmarking: The existing datadriven approach still encounters acceptance issues in the healthcare industry, as there is no clear consensus on whether such methods are reliable.

- Lack of optimization: Adoption of CNNs is more common in the literature due to their ability to detect critical features automatically without human supervision. However, it is computationally expensive and lacks the ability for the input data to be spatially invariant.

- Prone to outlier issue: The predictive model used to estimate statistical attributes from a temporal data sample is highly sensitive to outliers because the learning model corrects errors in the antecedents.

- Limited to specific design context: The modeling and implementation of existing data-driven schemes are limited to specific design contexts and may not provide similar performance when different contexts are introduced. The predictive ability of the existing learning models is restricted to the analysis of certain features. None of the research work is exhaustive in this manner; the clinical usefulness of the particular features used to derive these models must be considered.

Concurrently, it has been realized that most existing studies are still in the proof-of-concept stage, with small sample sizes and a lack of sufficient validation of prediction algorithms. Furthermore, the quantity and quality of the dataset limit the performance of the algorithm. It has been observed that current machine learning models are subjected to overfit issue, limited to theoretical validation, and suffers in readiness for clinical decision and implementation for effective patient care. Therefore, there is a need to develop an efficient scheme that can predict the true state of bipolar disorder, thereby facilitating the clinical decision-making process for treating patients without consuming too many computational resources and less prone to overfitting problems. The next section discusses the solution which is meant to address the research problem identified.

\section{RESEARCH METHODOLOGY}

The existing approaches to bipolar disorder are often prone to misdiagnosis, leading to difficulties in treating patients. The prime reason for choosing the proposed system is that an effective diagnosis of bipolar disorder has yet no scientific benchmarked model with higher success rate. Available solutions do exist but they are associated with limitation. Out of various problems in existing system, the proposed system chooses to address the research problems highlighted in Section III. The prime aim of the proposed study is to evolve up with a novel computational approach of detecting the state of bipolar disorder based on the feature obtained from motor activity. The significant contribution of this paper is the development of the novel neural network model, which is inspired by the architecture of the decision tree. Unlike existing approaches, the study aims to provide a better decision-making system in diagnosing mood swings disorder with emotional highs and lows with low time complexity and reduced possibility of overfitting. The schematic architecture of the proposed system is depicted in Fig. 1.

The modeling of the entire system adopts a phase-wise implementation procedure. Firstly, exploratory analysis is carried out to understand the characteristics of the dataset and the requirements related to suitable preprocessing. Based on the analysis, the dataset is temporal data as it consists of both time-series and special attributes. It has also been observed that the dataset contains details of 55 individuals, and among them, 23 are diagnosed with bipolar disorder. Further, preprocessing operation is carried out to execute treatment of missing data. In this process, median imputation is employed to handle the missing data. Afterward, descriptive statistics and curve fitting method-based correlation analysis are carried out from the viewpoint of the feature engineering task. In this phase, an analysis is performed regarding a MADRS score (i.e., clinician-rated scale) indicating the depression level. Based on the feature analysis, a new feature descriptor is created named delta-MADRS to analyze the severity of bipolar disorder in the patient. On the other hand, the obtained features are vectorized using a one-hot encoding technique to make the dataset fit to the proposed learning model to predict the actual state of bipolar disorder [30]. The proposed learning design adopts recurrent characteristics and a decision tree structure to better generalize the pattern from training samples that consist of both time-series and discrete observations.

The above Fig. 2 highlights the methodology of the implementation which is carried out in python environment. It is to be noted that the adopted dataset is not an image but it consist of various information in the form of text and numbers. After giving the patient meta-data information as an input in python script, the program access the time-series data which further consist of condition folder and control folder. Condition folder consists of time series data of the people where a doctor 
has diagnosed with bipolar disorder while control folder consists of time series data of the people who are in control group. Control group is those people where doctor has confirmed that they don't have bipolar disorder. This is further followed up by extracting MARD scores which is further subjected to correlation analysis for all the columns of data in MARDS score table. Categorical values are evaluated and machine learning approach is applied. This success rate is assessed by the multiple parameters of accuracy analysis explained in result section of this paper.

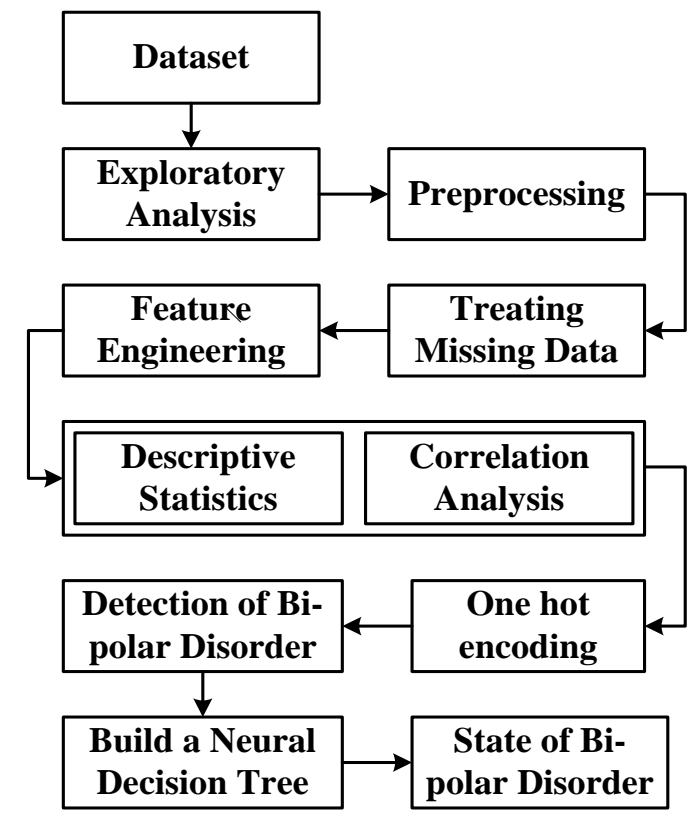

Fig. 1. Schematic Architecture of Proposed System.

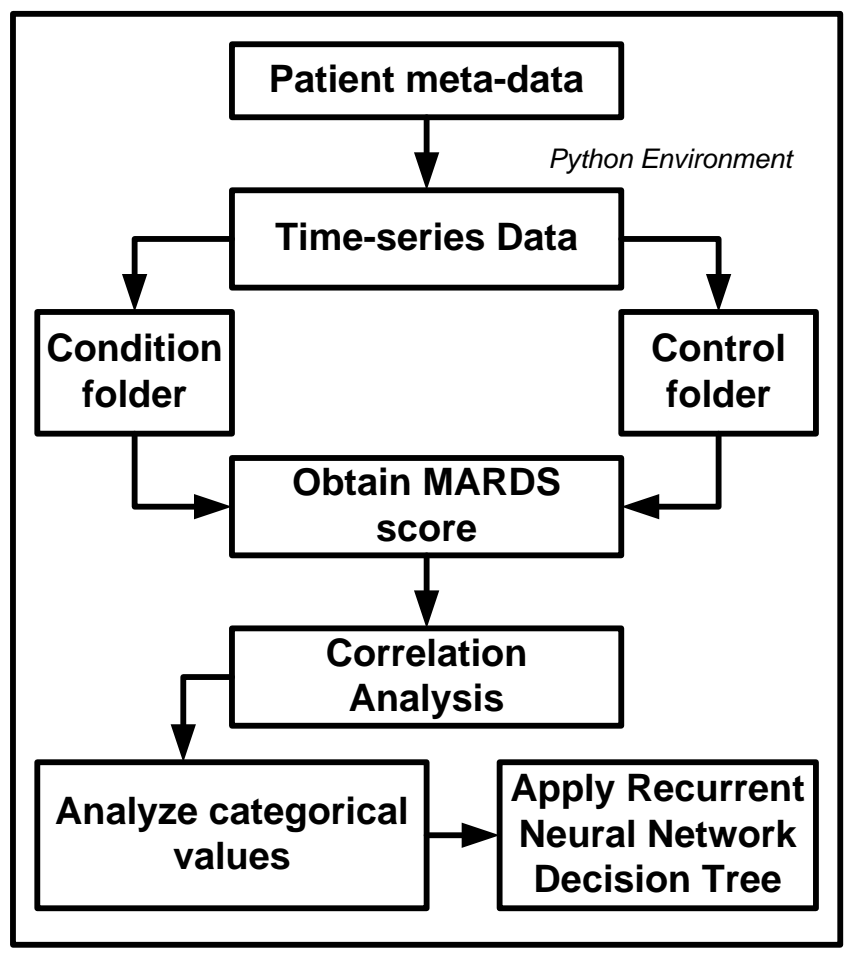

Fig. 2. Methodology for Implementation.

\section{SYSTEM IMPLEMENTATION}

The prime target of the proposed system design is to carry out a prediction for the subsequent state of bipolar disorder for the given dataset. This section discusses the algorithm implementation and its respective design execution.

\section{A. Algorithm for Prediction}

The core purpose of this algorithm is mainly to carry out a prediction of the subsequent state of bipolar disorder. The core basis of this algorithm is that unpredictable mood is quite challenging to be confirmed as a bipolar disorder. The majority of the existing schemes refer to depression as the indicator of bipolar disorder; however, it is unlikely to confirm this notion. The rationale is that depression could be a state of inactivity, too, and this cannot be concluded as a real state of bipolar disorder. Therefore, the proposed algorithm contributes towards offering a solution to this problem by offering an Actigraph-based monitoring system. This potentially assists in recording the different variants of motor activity of an individual. As the dataset used for the proposed system is available in time series, the prediction could be simplified by applying a unique machine learning approach. The steps of the proposed algorithm are as follows:

\begin{tabular}{l}
\hline Algorithm for Predicting State of Bipolar Disorder \\
\hline Input: $n$ (elements of the dataset) \\
Output: Pred (predicted outcome) \\
Start \\
1. For $i=1: n$ \\
2. If $n==e r r$ \\
3. $n \leftarrow \mathrm{s}_{\mathrm{val}}$ \\
4. $M=f_{1}(n)$ \\
5. $C_{\mathrm{val}}=f_{2}(\mathrm{M})$ \\
6. Pred=f $f_{3}\left(C_{\mathrm{val}}\right)$ \\
7. End \\
8. End \\
End
\end{tabular}

The prime dependency of the algorithm mentioned above is basically a numerical score obtained from MADRS, which is a standard score of rating depression of the subject at the time of observation. This score also assists in offering various statistical numerical values in the form of descriptive analysis. The dataset is preliminarily subjected to preprocessing, followed by the correlation analysis of numerical values. The algorithm implements feature engineering over the data followed by data correlation analysis. The algorithm then finds out the relevance of all the newly obtained data, followed by selecting the subject with reported bipolar disorder. All the time-series data is then loaded, followed by applying a machine learning approach to facilitate the prediction of bipolar disorder. The contribution of this algorithm are viz. i) with a reduced number of iterative operations, the algorithm assists in deploying machine learning operation, ii) the preprocessing carried out offers better elimination of all possible artifacts, iii) a unique feature engineering process is implemented towards obtaining a reliable numerical score of identifying an accurate state of prediction of bipolar disorder. The discussion of algorithmic steps is as follows: 


\section{B. Design of Algorithm Execution}

This section discusses the step-wise execution of the proposed algorithm that considers a dataset with $n$ elements (Line-1). The proposed system considers the presence of errors err within the dataset (Line-2). The algorithm considers the substitution of the errors within the dataset with a substituted value $s_{\text {val }}$ (Line-3). The three clauses used for data cleaning purposes are:

- For all the missing data among strings, it is filled with a definitive string.

- For all the missing data among integers, be it discrete or continuous, the empty columns are filled with the median value.

- All categorical data is converted to integers using the ONE HOT encoding technique.

The significance of using a function $f_{1}(\mathrm{x})$ by considering all the elements $n$ of the dataset, which finally leads to the construction of a new matrix $M$ (Line-4). The basic idea is to observe the changes appearing within the MADRS score. The advantage of performing this operation is obtaining a standard statistical numerical score that can offer a trend of positive depression ratings. However, there is still a possibility of complexity in the inference obtained from this statistical score in order to understand their connectivity concerning relevance. Therefore, the proposed system carries out correlation analysis using an explicit function $f_{2}(\mathrm{x})$. Its significance is that it can carry out and assists in graphical-based analysis is further used to find the correlation (Line-5). It is to be noted that this correlation analysis is carried out to assess the relevancy of the MADRS scores with respect to all the categorical values.

The final step of implementing the proposed study is basically applying a machine learning scheme, and a function $f_{3}(\mathrm{x})$ is developed for this purpose (Line-6). The significance of this function is that the proposed system follows the principle of decision tree architecture. The decision tree's applicability is high in the presence of a maximum number of discrete values compared to the continuous values, which is the pertaining case of the considered dataset. Therefore, the applicability of the decision tree is quite higher in the presence of computed correlated values $\mathrm{C}_{\mathrm{val}}$, The significance of this parameter is that it leads to the predicted outcome of Pred (Line-6). As the dataset consists of both time series and discrete values, a novel ANN known as Recurrent Neural decision tree is being proposed.

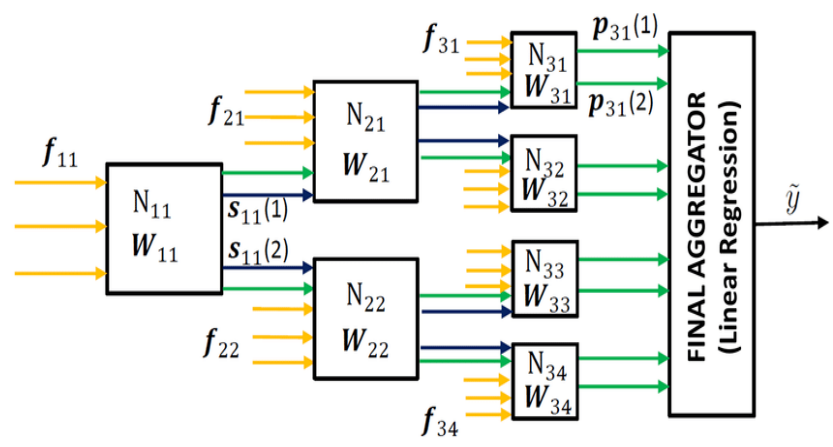

Fig. 3. Architecture of Proposed RNDT.
The architecture exhibited in Fig. 3 exhibits that every node in the decision tree is replaced with 3 neurons which will decide whether the activation should flow towards the right or left. It also shows the presence of input neurons $\mathrm{N}_{11}$, which is further split to $\mathrm{N}_{21}$ and $\mathrm{N}_{22}$. Further, the neuron node $\mathrm{N}_{21}$ is splitted to $N_{31}$ and $N_{32}$ while neuron node $N_{22}$ is split to $N_{33}$ and $\mathrm{N}_{34}$. Further, a different set of individual weights $\mathrm{W}_{11}, \mathrm{~W}_{21}$, $\mathrm{W}_{22}, \ldots$ is applied during every processing. This decides the category of the input. However, since this is a regression problem, the output is a continuous value instead of a single value. There is a presence of an aggregator module at the end that uses linear regression to get the continued value of predicted value $\mathrm{P}_{31}(1), \mathrm{P}_{31}(2), \ldots$.etc. The suitability of linear regression is quite high it can fine-tune the decision trees in case of regression.

\section{ANALYSIS}

From the implementation of the proposed scheme discussed in prior section, it is seen that proposed system make use of a simplified identification techniques on the basis of standard scale using psychometric properties. The adopted scheme is found to offer better consistency in its internal operation to ensure the reliability of this scale with higher acceptance. From the performance of trust factor of contents, the proposed scheme ensures that they must be directly linked with core concept of depression. The proposed system make use of different statistical variants of MADRS which is deployed for the purpose of assessing the efficacy of antidepressant trials since long time. The complete analysis is based on the data which is subjected to this scale for evaluating the depressive symptomology which is quite significant indicator for the patient undergoing antidepressant therapies. It doesn't consider any form of somatic symptoms but it essentially emphasizes over psychological symptoms associated with depression which is characterized by pessimistic thoughts, tension, and sadness. The next section discusses about the result being accomplished while the clinical inference of this usage of proposed system can be analyzed by adopting standards of this scale. The proposed system uses a scale that compromises of ten different items with lower and higher value of scale being 0 and 6 respectively. With accomplishment of higher score, the patient can be declared to be in depressive mode in higher degree. If the cumulative value of the score reside in between 0 and 6 than the patient is considered lack of depression symptoms, if the score is between 7 and 19 than it represents milder degree of depression, if the score is between 20 and 34 than it indicates moderate depression, while the greater score of 35 is considered to have severe depression. The critical case of depression is said to occur when this score is more than or equal to 60 .

Therefore, for an effective analysis with respect to granularity, it is essential to obtain the descriptive analysis of this score where score effectiveness can be assessed with respect to different variant of error observation (mean, root mean, and absolute). This inference is used as a back end logic to assess the proposed computational model in order to investigate the degree of bipolar disorder in a patient. The section discusses this with obtain numerical and graphical outcomes. 


\section{RESULT ANALYSIS}

This section discusses the results obtained from implementing the algorithm elaborated in the prior section. As stated in the prior section, that algorithm tends to predict the state of action as the prime indicator of the depression level of the subject. The illustration of this section is discussed with respect to the environment that is considered for an assessment, highlighting the results being achieved and a discussion of the results.

\section{A. Assessment Environment}

The development of the proposed system is carried out in a Python environment considering a normal windows machine. The implementation is carried out on the depression dataset [31]. The dataset consists of a metadata file as well as two forms of data, i.e., conditional data and controlled data. The former one is a time-series data of the subject who is positively diagnosed with a bipolar disorder by the doctor, while the latter one is also a time-series data that are negatively confirmed to have a bipolar disorder. The next part of the assessment environment is about using MADRS. As bipolar disorder represents a high amount of mood swings, the difference in the MADRS at the beginning of the observation is much different from that during the beginning. The measure of difference also indicates the severity of bipolar disorder as well. Hence a new column called $\triangle \mathrm{MADRS}$ is created, which is the difference between the MADRS1 and MADRS2.

\section{B. Results Obtained}

In order to carry out an effective analysis, the proposed system is compared with the conventional decision tree with respect to R2 score, Mean Squared Error (MSE), Root Mean Squared Error (RMSE), and Mean Absolute Error (MAE) as exhibited in Fig. 4 to Fig. 7, respectively. The outcome eventually showcased that the proposed Neural Decision Tree system offers better performance than the conventional decision tree.

TABLE I. DESCRIPTIVE ANALysis TABULATION

\begin{tabular}{|l|l|l|l|l|}
\hline Item & DAYS & MADRS1 & MADRS2 & $\Delta$ MADRAS \\
\hline count & 23 & 23 & 23 & 23 \\
\hline mean & 12.65217 & 22.73913 & 20 & -2.73910435 \\
\hline Std & 2.773391 & 4.797892 & 4.729021 & 3.968253466 \\
\hline Min & 5 & 13 & 11 & -13 \\
\hline $25 \%$ & 12.5 & 18.5 & 16 & -5 \\
\hline $50 \%$ & 13 & 24 & 21 & -3 \\
\hline $75 \%$ & 14 & 26 & 24.5 & 0 \\
\hline Max & 18 & 29 & 28 & 4 \\
\hline
\end{tabular}

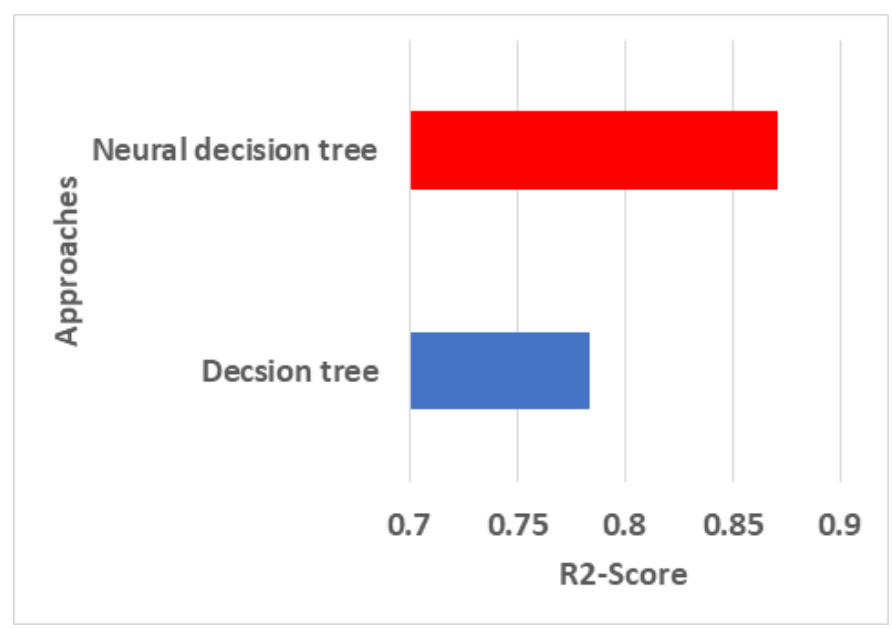

Fig. 4. Comparative Analysis of R2 Score.

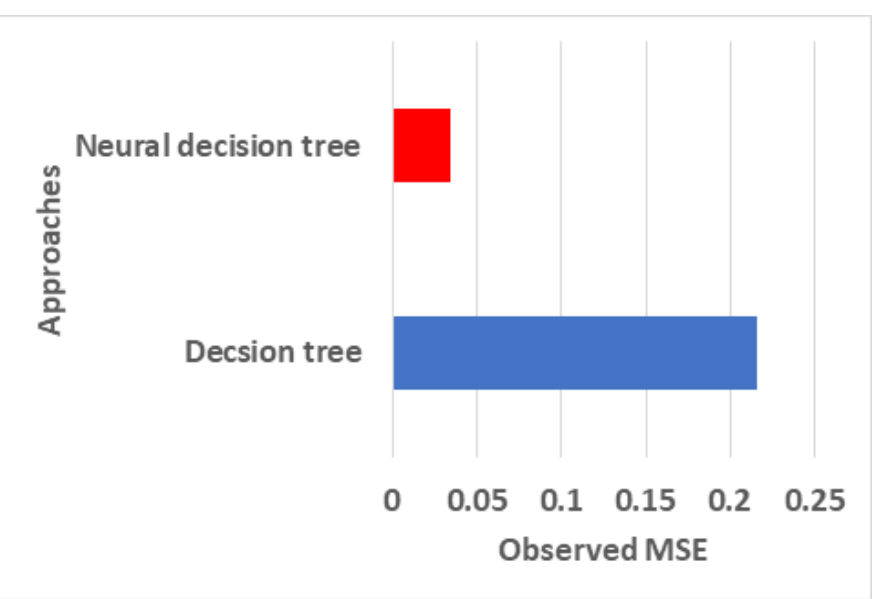

Fig. 5. Comparative Analysis of MSE.

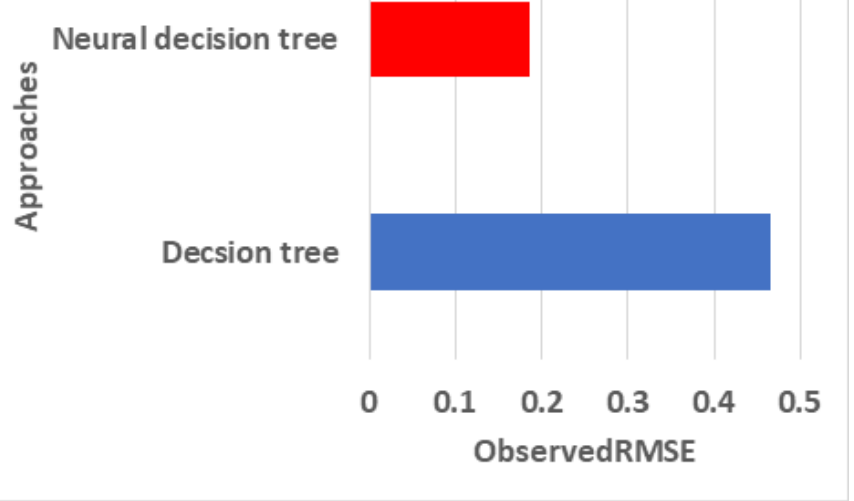

Fig. 6. Comparative Analysis of RMSE. 


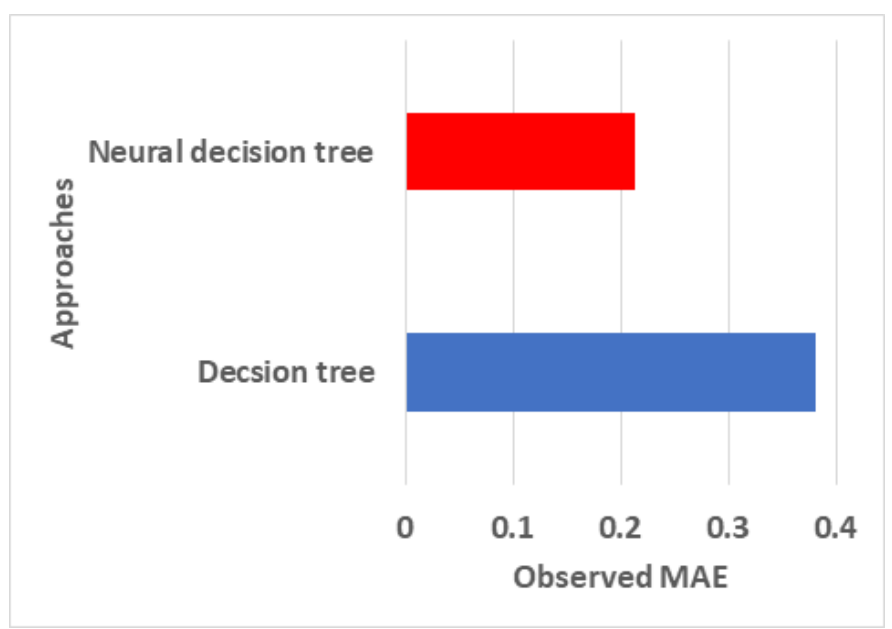

Fig. 7. Comparative Analysis of MAE.

\section{Result Discussion}

The analysis of the proposed system's outcome initiates from analyzing the MARD score. The numerical outcome exhibited in Table I clearly indicates that more than $75 \%$ of the diagnosed people with the MARDS score deteriorated during the observation. The outcome eventually indicates that the MADRS score reduces up after therapy compared to the prior MADRS score before therapy. The next set of outcomes is obtained from the correlation analysis for all the numerical values in Table I. The outcome indicates that the MARDS1 and 2 are correlated to each other. The above graphs use the curve fitting method to indicate the correlation. Based on these, more conclusive remarks can be obtained over the dataset as follows:

- Age: People with age-group of 45-49 as well as 35-39 are more prone to depression.

- Gender: Women are found to be more susceptible for bipolar disorder compared to man.

- Type of Bipolar Disorder: Type-2 bipolar disorder is found to be more predominant in contrast to any other type.

- Mood Swings: The analysis found that the patient often does not indicate sadness, making the diagnosis harder. The bipolar disorder only means frequent mood swings but not prolonged sadness. Due to these mood swings, it becomes difficult to predict the behaviors of the patient. This proves the scope of the study that since it is difficult to predict, a machine-learning algorithm has been used to help the therapist.

- Type of Admission: Analysis shows that bipolar patients are seldom admitted to the hospital most of the time; they are outpatients who can be treated like normal patients for any other disorder.

- Education: The lesser the person is educated, the more likely the person gets bipolar disorder.

- Marriage: Bipolar disorder has nothing to do with marital status.

\section{CONCLUSION}

There have been various myths about a proper diagnosis of a patient with bipolar disorder. In various analyses, it is a myth to associate sadness or a specific state of mind to bipolar disorder. The limiting factor of this paper could be that it doesn't consider any form of somatic symptoms as it uses MADRS scheme. It doesn't affect the outcome as the proposed system contributes towards connecting activity-based state with depressive scale as a novel approach. However, the proposed system proves that adopting an activity-based state is the precise means to perform an analysis. The novelty introduced by the proposed study is as follows:

- Unlike any existing studies on bipolar disorder, the proposed scheme doesn't directly consider the dataset as it is rather, it performs a set of operations to make it more suitable for analysis,

- The proposed system makes use of Actigraph for considering the motor activity of a subject, thereby considering time-series based data suitable for predictive analysis,

- The complete analysis is carried out considering all the meta-data present in the dataset with respect to days of observation, age, type of diagnosis, melanch, type of admission, marriage, employment, etc.,

- The proposed system introduces three sequential processes for data cleaning in order to find all the missing data as well as the transformation of the categorical data,

- The model harnesses the standard scale of MADRS score for accounting for the fluctuation in the mood swing by exploring the difference in the individual progressive scores, vi) contextual accuracy in the data inference is obtained by applying contextual analysis,

- Usage of a recurrent decision tree that hybridizes a recurrent neural network with a decision tree for making precise predictive outcomes for bipolar disorder.

The quantified outcome of the proposed study are as follows: The proposed scheme offers approximately $50 \%$ of betterment in R2 score compared to conventional decision trees, approximately $80 \%$ reduced value of MSE, $62 \%$ reduced value of RMSE, and $41 \%$ reduction of MAE in comparison to conventional decision tree approach. Hence adoption of proposed neural decision tree significantly assists in modelling various collective activity-based data with each pattern arranged in form of decision tree with less dependency. This significantly assists in achieving faster as well as simpler computational efficiency while executing the model.

The future work of the proposed study could be in direction of further optimizing the model with more constraint inclusion. This could be further achieved by non-linear constraint modelling where along with activity, various other non-linear non-activity based parameter could be opted. The future work would also be in direction towards inclusion of multi-objective function to cater up cost effective modelling demands. 


\section{REFERENCES}

[1] Paris, Joel. "Mood disorders and personality disorders: Simplicity and complexity." In Borderline Personality and Mood Disorders, pp. 3-9. Springer, New York, NY, 2015.

[2] Singh, Meharban. "Compulsive digital gaming: an emerging mental health disorder in children." The Indian Journal of Pediatrics 86, no. 2 (2019): 171-173.

[3] Blackwood, Douglas HR, Ben J. Pickard, Pippa A. Thomson, Kathryn L. Evans, David J. Porteous, and Walter J. Muir. "Are some genetic risk factors common to schizophrenia, bipolar disorder and depression? Evidence from DISC1, GRIK4 and NRG1." Neurotoxicity Research, vol.11, no. 1, pp.73-83, 2007.

[4] Gilkes, Melissa, T. Perich, and T. Meade. "Predictors of self-stigma in bipolar disorder: Depression, mania, and perceived cognitive function." Stigma and Health, vol.4, no. 3, 2019.

[5] Tondo, Leonardo, Gustavo H Vazquez, and Ross J Baldessarini. "Depression and mania in bipolar disorder." Current neuropharmacology, vol.15, no. 3, pp.353-358, 2017.

[6] Koukopoulos, A., Reginaldi, D., Tondo, L., Visioli, C. and Baldessarini, R.J., "Course sequences in bipolar disorder: depressions preceding or following manias or hypomanias." Journal of affective disorders, vol.151, Iss.(1), pp.105-110, 2013.

[7] Yashaswini, K. A., and Shreyas Rao. "Bipolar Disorder: A Pathway Towards Research Progress in Identification and Classification." In Computer Science On-line Conference, pp. 205-214. Springer, Cham, 2020.

[8] Yashaswini K.A., Saxena A.K., "Novel Classification Modelling for Bipolar Disorder Using Non-verbal Attributes for Classification". In: Venugopal K.R., Shenoy P.D., Buyya R., Patnaik L.M., Iyengar S.S. (eds) Data Science and Computational Intelligence. ICInPro 2021. Communications in Computer and Information Science, vol 1483. Springer, Cham., 2021 https://doi.org/10.1007/978-3-030-91244-4_19.

[9] Vasu, V. and Indiramma, M., 2020, September. “A Survey on Bipolar Disorder Classification Methodologies using Machine Learning”. IEEE International Conference on Smart Electronics and Communication, pp. 335-340, 2020.

[10] Phillips, M.L. and Kupfer, D.J., "Bipolar disorder diagnosis: challenges and future directions", The Lancet, vol.381, Iss.9878, pp.1663-1671, 2013.

[11] Ceccarelli, F., Mahmoud, M. Multimodal temporal machine learning for bipolar disorder and Depression Recognition. Pattern Anal Applic, ISSN 1433-754, 2021. DOI: https://doi.org/10.1007/s10044-021-01001-y.

[12] Sánchez-Morla, E.M., Fuentes, J.L., Miguel-Jiménez, J.M., Boquete, L., Ortiz, M., Orduna, E., Satue, M. and Garcia-Martin, E., "Automatic Diagnosis of Bipolar Disorder Using Optical Coherence Tomography Data and Artificial Intelligence", Journal of Personalized Medicine, vol.11, Iss.(8), p.803, 2021.

[13] Mateo-Sotos, J., Torres, A.M., Santos, J.L. et al., “A Machine LearningBased Method to Identify Bipolar Disorder Patients". Circuits Syst Signal Process, 2021. https://doi.org/10.1007/s00034-021-01889-1.

[14] Chen, ZhiHong, T. Yan, E. Wang, H. Jiang, Y. Tang, X. Yu, J. Zhang, and C. Liu. "Detecting abnormal brain regions in schizophrenia using structural MRI via machine learning." Computational intelligence and neuroscience, 2020.

[15] S. Qiu, Q. Yue, F. Zhu, and K. Shu. "The Identification research of bipolar disorder based on CNN." In Journal of Physics: Conference Series, IOP Publishing, vol.1168, no. 3, p. 032125, 2019.

[16] Li, Z., Li, W., Wei, Y., Gui, G., Zhang, R., Liu, H., Chen, Y. and Jiang, Y., "Deep learning based automatic diagnosis of first-episode psychosis, bipolar disorder and healthy controls". Computerized Medical Imaging and Graphics, 89, p.101882, 2021.
[17] Huang, Kun-Yi, Chung-Hsien Wu, and Ming-Hsiang Su. "Attentionbased convolutional neural network and long short-term memory for short-term detection of mood disorders based on elicited speech responses." Pattern Recognition, vol.88, pp.668-678, 2019.

[18] Du, Z., Li, W., Huang, D. and Wang, Y., 2018, October. Bipolar disorder recognition via multi-scale discriminative audio temporal representation. In Proceedings of the 2018 on Audio/Visual Emotion Challenge and Workshop (pp. 23-30), 2018.

[19] T. Matsubara, T. Tashiro and K. Uehara, "Deep Neural Generative Model of Functional MRI Images for Psychiatric Disorder Diagnosis," in IEEE Transactions on Biomedical Engineering, vol. 66, no. 10, pp. 2768-2779, Oct. 2019, doi: 10.1109/TBME.2019.2895663.

[20] O. Cigdem et al., "Effects of Covariates on Classification of Bipolar Disorder Using Structural MRI," Scientific Meeting on ElectricalElectronics \& Biomedical Engineering and Computer Science (EBBT), 2019, pp. 1-4, doi: 10.1109/EBBT.2019.8741586.

[21] D. Fitriati, F. Maspiyanti and F. A. Devianty, "Early Detection Application of Bipolar Disorders Using Backpropagation Algorithm," 6th International Conference on Electrical Engineering, Computer Science and Informatics (EECSI), pp. 40-44, 2019, doi: 10.23919/EECSI48112.2019.8977102.

[22] G. Castro et al., "Applying Association Rules to Study Bipolar Disorder and Premenstrual Dysphoric Disorder Comorbidity," IEEE Canadian Conference on Electrical \& Computer Engineering (CCECE), pp. 1-4, 2018 doi: 10.1109/CCECE.2018.8447747.

[23] P. C. Büttenbender, E. G. d. A. Neto, W. F. Heckler and J. L. V. Barbosa, "A computational model for identifying behavioral patterns in people with neuropsychiatric disorders," in IEEE Latin America Transactions, vol. 20, no. 4, pp. 582-589, April 2022, doi: 10.1109/TLA.2022.9675463.

[24] L. C. Nunes, P. R. Pinheiro, M. C. Dantas Pinheiro, M. Simão Filho, R. E. Comin Nunes and P. G. C. Dantas Pinheiro, "Automatic Detection and Diagnosis of Neurologic Diseases," in IEEE Access, vol. 7, pp. 29924-29941, 2019, doi: 10.1109/ACCESS.2019.2899216.

[25] C. -Y. Lee, J. -H. Zeng, S. -Y. Lee, R. -B. Lu and P. -H. Kuo, "SNP Data Science for Classification of Bipolar Disorder I and Bipolar Disorder II," in IEEE/ACM Transactions on Computational Biology and Bioinformatics, vol. 18, no. 6, pp. 2862-2869, 1 Nov.-Dec. 2021, doi: 10.1109/TCBB.2020.2988024.

[26] H. G. Villasanti and K. M. Passino, "Modeling and Analysis of Mood Dynamics in the Bipolar Spectrum," in IEEE Transactions on Computational Social Systems, vol. 7, no. 6, pp. 1335-1344, Dec. 2020, doi: 10.1109/TCSS.2020.3028205.

[27] K. -Y. Huang, C. -H. Wu, M. -H. Su and Y. -T. Kuo, "Detecting Unipolar and Bipolar Depressive Disorders from Elicited Speech Responses Using Latent Affective Structure Model," in IEEE Transactions on Affective Computing, vol. 11, no. 3, pp. 393-404, 1 July-Sept. 2020, doi: 10.1109/TAFFC.2018.2803178.

[28] A. Demir, M. Özkan and A. M. Uluğ, "A Macro-Structural Dispersion Characteristic of Brain White Matter and Its Application to Bipolar Disorder," in IEEE Transactions on Biomedical Engineering, vol. 68, no. 2, pp. 428-435, Feb. 2021, doi: 10.1109/TBME.2020.3002688.

[29] Kumar, Yashaswini Kunjali Ajeeth, and Aditya Kishore Saxena. "Stochastic modelling of transition dynamic of mixed mood episodes in bipolar disorder." International Journal of Electrical \& Computer Engineering vol.12, pp.2088-8708, no. 1, 2022.

[30] Y. H. Liu, Python Machine Learning By Example, Packt Publishing, ISBN: 9781783553129, 178355312X, 2017.

[31] A. Jan, H. Meng, Y. F. B. A. Gaus and F. Zhang, "Artificial Intelligent System for Automatic Depression Level Analysis Through Visual and Vocal Expressions," in IEEE Transactions on Cognitive and Developmental Systems, vol. 10, no. 3, pp. 668-680, Sept. 2018, doi: 10.1109/TCDS.2017.2721552. 